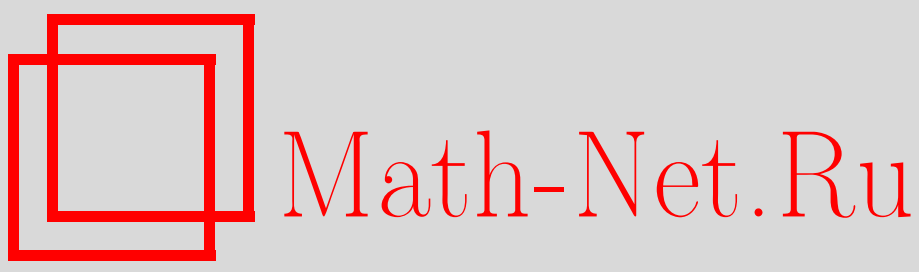

В. С. Климов, Усреднение параболических включений, Матем. сб., 2004, том 195, номер 1, 21-36

DOI: https://doi.org/10.4213/sm791

Использование Общероссийского математического портала Math-Net.Ru подразумевает, что вы прочитали и согласны с пользовательским соглашением

http://www . mathnet.ru/rus/agreement

Параметры загрузки:

IP : 54.196 .121 .252

26 апреля 2023 г., 09:35:52 
УДК 517.958

\author{
В. С. Климов
}

\title{
Усреднение параболических включений
}

\begin{abstract}
Устанавливается вариант первой теоремы Боголюбова для бесконечномерных дифференциальных включений. Формулируются достаточные условия асимптотической устойчивости нулевого решения параболического включения с однородной и нестационарной главной частью.

Библиография: 22 названия.
\end{abstract}

\section{Введение}

В работе устанавливается вариант первой теоремы Боголюбова для бесконечномерных дифференциальных включений параболического типа. Приводятся достаточные условия асимптотической устойчивости нулевого решения параболического включения с однородной (степени $m \geqslant 1$ ) по фазовому переменному и нестационарной по времени главной частью. Анализируется зависимость асимптотики нетривиальных решений от степени однородности. Как полагает автор, результаты, относящиеся к случаю $m>1$, являются новыми и для дифференциальных уравнений в конечномерњых пространствах.

Используются следуюшие обозначения: $\|x ; E\|=\|x\|_{E}$ - норма элемента $x$ в $B$-пространстве $E, E^{*}$ - сопряженное к $E$ пространство, $\left(x, x^{*}\right)$ - значение функционала $x^{*}$ из $E^{*}$ на элементе $x \in E, \sigma\left(E, E^{*}\right)$ и $\sigma\left(E^{*}, E\right)$ - слабые топологии на $E$ и $E^{*}$, порождаемые формой $(\cdot, \cdot), s\left(x^{*}, \mathscr{D}\right)=\sup \left\{\left(x, x^{*}\right), x \in \mathscr{D}\right\}$, $x^{*} \in E^{*},-$ опорная функция множества $\mathscr{D} \subset E,\|\mathscr{D}\|_{E}=\sup \left\{\|x\|_{E}, x \in \mathscr{D}\right\}$, $\theta_{E}\left(\mathscr{D}_{1}, \mathscr{D}_{2}\right)=\sup _{x_{1} \in \mathscr{D}_{1}} \inf _{x_{2} \in \mathscr{D}_{2}}\left\|x_{1}-x_{2}\right\|_{E}-$ уклонение множества $\mathscr{D}_{1} \subset E$ от множества $\mathscr{D}_{2} \subset E, L^{p}(T, E)(C(T, E))$ - пространство функций, измеримых по Лебегу-Бохнеру (непрерывных) на отрезке $T \subset \mathbb{R}$ со значениями в $E$ (как обычно, эквивалентные функции отождествляются), нормы в $L^{p}(T, E), 1 \leqslant p \leqslant \infty$, и $C(T, E)$ определяются стандартным образом [1]; символы $\mathscr{B}(E),(\mathrm{Cv}(E))$ обозначают совокупность непустых ограниченных (замкнутых и выпуклых) подмножеств пространства $E$. Все банаховы пространства рассматриваются над полем $\mathbb{R}$ действительных чисел. Если $E_{1}, E_{2}-B$-пространства, то запись $E_{1} \rightarrow E_{2}\left(E_{1} \stackrel{k}{\longrightarrow} E_{2}\right)$ означает, что $E_{1}$ непрерывно (компактно) вложено в $E_{2} ; E_{1} \times E_{2}-$ прямое произведение $E_{1}$ и $E_{2}$; если $E_{1}, E_{2}$ вложены в линейное топологическое пространство $E$, то с $E_{1}, E_{2}$ связаны $B$-пространства $E_{1} \cap E_{2}$ и $E_{1}+E_{2}$ [1; гл. 1$]$.

\section{§1. Специальные классы многозначных отображений}

Многозначное отображение (м-отображение) $F$ множества $\mathscr{D}_{1}$ в множество $\mathscr{D}_{2}$ - это оператор, ставящий в соответствие элементу $v$ из $\mathscr{D}_{1}$ непустое множество $F(v) \subset \mathscr{D}_{2} ;$ если $\mathscr{D} \subset \mathscr{D}_{1}$, то $F(\mathscr{D})=\bigcup_{v \in \mathscr{D}} F(v)$ - область значений отображения $F$ на множестве $\mathscr{D}$. М-отображение $F: \mathscr{D}_{1} \rightarrow \mathscr{D}_{2}\left(\mathscr{D}_{1}, \mathscr{D}_{2}-\right.$ подмножества $B$-пространств $\left.E_{1}, E_{2}\right)$ назьвают ограниченныцм, если $F(\mathscr{D}) \in \mathscr{B}\left(E_{2}\right)$ 
для каждого $\mathscr{D}$ из $\mathscr{B}\left(E_{1}\right), \mathscr{D} \subset \mathscr{D}_{1}$; отображение $F: E_{1} \rightarrow E_{2}$ хеминепрерывно сверху на $E_{1}$, если для каждого $x^{*}$ из $E_{2}^{*}$ функция $v \mapsto s\left(x^{*}, F(v)\right)$ полунепрерывна сверху на $E_{1}$; в случае $E_{1}=E_{2}^{*}$ отображение $F: E_{1} \rightarrow E_{2} p a-$ диально хеминепрерывно сверху, если при любых $v, w$ из $E_{1}$ справедливо неравенство $s(v, F(w)) \geqslant \overline{\lim }_{\lambda \rightarrow+0} s(v, F(w+\lambda v))$. Если образы м-отображения $F: E_{1} \rightarrow E_{2}$ принадлежат классу $\mathrm{Cv}\left(E_{2}\right)$, то будем записывать это следующим образом: $F: E_{1} \rightarrow \operatorname{Cv}\left(E_{2}\right)$. Без дополнительных пояснений используется терминология, принятая в теории измеримых м-отображений [2]-[8].

Пусть $E$ - рефлексивное $B$-пространство. М-отображение $F: E \rightarrow \mathrm{Cv}\left(E^{*}\right)$ назовем квазимонотонным, если из соотношений $u \in E, v \in E,\|u\|_{E} \leqslant R$, $\|v\|_{E} \leqslant R, u^{*} \in F(u), v^{*} \in F(v)$ вытекает неравенство

$$
\left(u-v, u^{*}-v^{*}\right) \geqslant-c\left(R,\|u-v\|_{E}\right),
$$

где $c(R, \cdot)$ - непрерьвная неотрицательная функция, $c(R, \xi)=o(\xi)$ при $\xi \rightarrow+0$. В случае $c(R, \xi) \equiv 0 F$-монотонное отображение.

Лемма 1. Пусть $F: E \rightarrow \mathrm{Cv}\left(E^{*}\right)$ - ограниченное квазимонотонное радиально хеминепрерывное сверху м-отображение. Тогда м-отображение $F$ хеминепрерывно сверху.

ДокаЗАТЕЛьство. Пусть $y_{i} \rightarrow y$ в $E, w \in E$ и $\left(w, y_{i}^{*}\right)=s\left(w, F\left(y_{i}\right)\right), y_{i}^{*} \in$ $F\left(y_{i}\right)$. Без ограничения общности можно считать, что $y_{i}^{*} \rightarrow y^{*}$ в $\sigma\left(E^{*}, E\right)$. Зафиксируем $u$ из $E, u^{*}$ из $F(u)$ и число $R$ так, что $\left\|y_{i}\right\|_{E} \leqslant R,\|y\|_{E} \leqslant R$. В силу (1) справедливо неравенство $\left(y_{i}-u, y_{i}^{*}-u^{*}\right) \geqslant-c\left(R,\left\|y_{i}-u\right\|_{E}\right)$, переходящее при $i \rightarrow \infty$ в оценку $\left(y-u, y^{*}-u^{*}\right) \geqslant-c\left(R,\left\|y_{i}-u\right\|_{E}\right)$. Из этой оценки вытекает соотношение $s(u-y, F(u)) \geqslant\left(u-y, u^{*}\right)-c\left(R,\|y-u\|_{E}\right)$. Полагая $u=y+\lambda v$, $v \in E, \lambda>0$, имеем

$$
s(v, F(y+\lambda v)) \geqslant\left(v, y^{*}\right)-\frac{1}{\lambda} c\left(R, \lambda\|v\|_{E}\right) .
$$

Используя радиальную хеминепрерывность сверху отображения $F$, получаем при $\lambda \rightarrow+0$ неравенство $s(v, F(y)) \geqslant\left(v, y^{*}\right)$. Ввиду произвольности $v$ из $E$ отсюда вытекает включение $y^{*} \in F(y)$, в частности, $\left(w, y^{*}\right) \leqslant s(w, F(y))$. Таким образом,

$$
\lim _{i \rightarrow \infty} s\left(w, F\left(y_{i}\right)\right)=\lim _{i \rightarrow \infty}\left(w, y_{i}^{*}\right)=\left(w, y^{*}\right) \leqslant s(w, F(y))
$$

Последнее неравенство означает хеминепрерьвность сверху м-отображения $F$. Лемма доказана.

Для однозначных операторов близкие к лемме 1 утверждения хорошо известны (см. [1], [9]-[11]).

Пусть $E_{1}-B$-пространство, $E_{2}$ - сепарабельное рефлексивное $B$-пространство, $T=[\alpha, \beta] \subset \mathbb{R}$. Будем говорить, что м-отображение $\mathscr{G}: T \times E_{1} \rightarrow \mathrm{Cv}\left(E_{2}\right)$ удовлетворяет слабому условию Каратеодори, и писать $\mathscr{G} \in \mathbb{C}_{*}\left(T, E_{1}, E_{2}\right)$, если

1) м-отображение $t \mapsto \mathscr{G}(t, v)$ измеримо при любом $v$ из $E_{1}$;

2) почти при каждом $t$ из $T$ м-отображение $v \mapsto \mathscr{G}(t, v)$ хеминепрерывно;

$3)$ для каждого $R>0$ найдется такая функция $\varphi_{R}$ из $L^{1}(T)$, что почти при всех $t$ из $T$ и $\|v\|_{E_{1}} \leqslant R$ выполняется неравенство $\|\mathscr{G}(t, v)\|_{E_{2}} \leqslant \varphi_{R}(t)$. 
В этом случае отображение суперпозиционно измеримо, т.е. для любой измеримой функции $y: T \rightarrow E_{1}$ непусто множество $G(y)$ измеримых сечений $t \mapsto$ $\mathscr{G}(t, y(t))$. Действительно, если $y$ - счетнозначная функция, то непустота $G(y)$ следует из условия 1$)$. Для произвольной функции $y$ из $L^{\infty}(T, E)$ существует последовательность счетнозначных функций $y_{i}$, сходящаяся к $y$ в $L^{\infty}\left(T, E_{1}\right)$. Пусть $z_{i} \in G\left(y_{i}\right), i=1,2, \ldots$ В силу условия 3$)$ последовательность $z_{i}$ компактна в топологии $\sigma\left(L^{1}\left(T, E_{2}\right), L^{\infty}\left(T, E_{2}^{*}\right)\right)$, поэтому можно считать, что $z_{i} \rightarrow z$ в этой топологии. Повторяя рассуждения, проведенные в [5; гл. 3], можно установить включение $z \in G(y)$, т.е. $G(y) \neq \varnothing \forall y \in L^{\infty}\left(T, E_{1}\right)$. Отсюда без труда выводится непустота $G(y)$ для любой измеримой функции $y: T \rightarrow E_{1}$. Сохраним обозначение $G$ за сужением $G$ на банаховы пространства измеримых функций. Как и в случае однозначных операторов суперпозиции [12], оценка вида $\|\mathscr{G}(t, v)\|_{E_{2}} \leqslant k\|v\|_{E_{1}}^{p_{1}} p_{2}+\gamma(t)$, $v \in E_{1}, 1 \leqslant p_{1}, p_{2}<\infty, k>0, \gamma \in L^{p_{2}}(T)$, влечет оценку роста м-отображения $G: L^{p_{1}}\left(T, E_{1}\right) \rightarrow L^{p_{2}}\left(T, E_{2}\right)$.

Пусть $V$ - рефиексивное сепарабельное $B$-пространство с нормой $\|\cdot\|, H$ - гильбертово пространство с нормой $|\cdot|, V \stackrel{k}{\longrightarrow} H$ и $V$ плотно в $H$. Пространство $H$ отождествляется с сопряженным ему $H^{*}$, а $H^{*}=H$ - с некоторьм подпространством сопряженного к $V$ пространства $V^{*}$. Число $\left(v, v^{*}\right)$ означает и скалярное произведение элементов $v, v^{*}$ из $H$, и значение функционала $v^{*}$ на элементе $v \in V$.

Сопоставим отрезку $T=[\alpha, \beta] \subset \mathbb{R}$ и числам $p, p_{0}, 2 \leqslant p \leqslant p_{0}<\infty$, $B$-пространства $Y=L^{p}(T, V) \cap L^{p_{0}}(T, H), Z=L^{q}\left(T, V^{*}\right)+L^{q_{0}}(T, H)$, где $1 / p+1 / q=1,1 / p_{0}+1 / q_{0}=1$. Пространства $Y, Z$ рефилексивны и сепарабельны, $Z$ можно идентифицировать с сопряженньм к $Y$. Билинейная форма, задаюшая двойственность между $Y, Z$, определяется (см. [1; гл. 4]) равенством

$$
\langle y, z\rangle=\int_{T}(y(t), z(t)) d t
$$

Если необходимо подчеркнуть зависимость пространств $Y, Z$ от $T$, будем обозначать их символами $Y(T), Z(T)$.

Положим $W=\left\{y: y \in Y, y^{\prime} \in Z\right\},\|y\|_{W}=\|y\|_{Y}+\left\|y^{\prime}\right\|_{Z}$. Пространство $W$ непрерывно вложено в пространство $C(T, H)$; это позволяет ввести понятие значения функции $y$ из $W$ в каждой точке отрезка $T$. Если $y \in W$, то функция $t \mapsto|y(t)|^{2}$ абсолютно непрерывна на $T$ и справедлива формула $\left(|y(t)|^{2}\right)^{\prime}=2\left(y(t), y^{\prime}(t)\right)$ п.в. [1; гл. 4].

Лемма 2. Пусть $E-$ - В-пространство, $V \stackrel{k}{\longrightarrow} E \rightarrow H$. Тогда $W \stackrel{k}{\longrightarrow}$ $L^{p}(T, E) \cap L^{r}(T, H)$ для любого $r$ из $(1, \infty)$.

ДокАЗАтЕльство. Вложение $W \stackrel{k}{\longrightarrow} L^{p}(T, H)$ установлено в [11; гл. 1]. Так как $E \rightarrow H$, то $W \stackrel{k}{\longrightarrow} L^{p}(T, H)$. Компактное в $L^{p}(T, H)$ и ограниченное в $C(T, H)$ множество компактно в $L^{r}(T, H)$ при любом $r<\infty$. Теперь требуемый результат вытекает из вложения $W \rightarrow C(T, H)$.

Лемма доказана.

Ниже $W_{1}$ - реффлексивное $B$-пространство, $W \stackrel{k}{\longrightarrow} W_{1}, Y \rightarrow W_{1}$. Так как $Y \rightarrow$ $W_{1}$, то $W_{1}^{*} \rightarrow Y^{*}=Z$. В качестве $W_{1}$ можно взять, например, $W_{1}=L^{p}(T, E) \cap$ $L^{r}(T, H)$, где $E$ - рефлексивное пространство, $V \stackrel{k}{\longrightarrow} E \rightarrow H, 1<r<\infty$; в 
этом случае $W_{1}^{*}$ можно идентифицировать с пространством $L^{q}\left(T, E^{*}\right)+L^{s}(T, H)$, $1 / r+1 / s=1$.

Возврашаясь к обшему случаю, назовем последовательность $\xi_{i}$ из $Z$ полусходящейся, если она представима в виде $\xi_{i}=f_{i}+g_{i}$, где $f_{i} \rightarrow f$ в $Z, g_{i} \rightarrow g$ в $\sigma\left(W_{1}^{*}, W_{1}\right)$. Будем писать $\xi_{i} \rightarrow \xi=f+g$.

ЛЕмма 3. Пусть $y_{i}$ - ограниченная в пространстве $W$ последовательность, $y_{i} \rightarrow$ в в $\sigma(Y, Z), \xi_{i} \in Z$ и $\xi_{i} \rightarrow \xi$. Тогда $\left(y_{i}, \xi_{i}\right) \rightarrow(y, \xi)$ в $\sigma\left(L^{1}, L^{\infty}\right)$.

ДОкаЗАТЕЛЬСТво. Пусть $\xi_{i}=f_{i}+g_{i}, i=1,2, \ldots, f_{i} \rightarrow f$ в $Z, g_{i} \rightarrow g$ в $\sigma\left(W_{1}^{*}, W_{1}\right)$. Так как $y_{i}$ - ограниченная в пространстве $W$ последовательность и $y_{i} \rightarrow y$ в $\sigma(Y, Z)$, то $y_{i} \rightarrow y$ в пространстве $W_{1}$. Из отмеченных свойств последовательностей $f_{i}, g_{i}, y_{i}$ вытекают соотношения $\left\langle 1_{\Delta} y_{i}, 1_{\Delta} f_{i}\right\rangle \rightarrow\left\langle 1_{\Delta} y, 1_{\Delta} f\right\rangle$, $\left\langle 1_{\Delta} y_{i}, 1_{\Delta} g_{i}\right\rangle \rightarrow\left\langle 1_{\Delta} y, 1_{\Delta} g\right\rangle$, здесь $1_{\Delta}-$ характеристическая функция измеримого множества $\Delta \in T$. В силу произвольности выбора $\Delta$ отсюда следует сходимость $\left(y_{i}, \xi_{i}\right) \rightarrow(y, \xi)$ в $\sigma\left(L^{1}, L^{\infty}\right)$. Лемма доказана.

М-отображение $F: Y(T) \rightarrow Z(T)$ назовем локальным, если сушествует порождающее его м-отображение $\mathscr{F}: T \times V \rightarrow V^{*}$ класса $\mathbb{C}_{*}\left(T, V, V^{*}\right)$. Для любого $y$ из $Y(T)$ множество $F(y)$ совпадает с множеством измеримых сечений отображения $t \mapsto \mathscr{F}(t, y(t))$. Для локального м-отображения $F: Y(T) \rightarrow Z(T)$ естественньм образом определяется его сужение на пространство $Y(J)$, где $J$ - отрезок, содержащийся в отрезке $T$.

\section{§2. Сходимость последовательности решений задачи Коши}

Пусть $T=[\alpha, \beta], F: Y(T) \rightarrow Z(T)$ - м-отображение пространства $Y(T)$ в пространство $Z(T)$. Решением дифференциального включения $0 \in y^{\prime}+F(y)$ называется функция $y$ класса $W$, для которой $-y^{\prime} \in F(y)$; если при этом $y(\alpha)=h$, то $y$ - решение задачи Коши

$$
0 \in y^{\prime}+F(y), \quad y(\alpha)=h
$$

Введем последовательность м-отображений $F_{i}: Y(T) \rightarrow Z(T), i=0,1, \ldots$, удовлетворяющих следующим условиям:

$\left.\mathbb{A}_{1}\right) \bigcup_{i} F_{i}(M) \subset \mathscr{B}(Z(T)) \forall M \in \mathscr{B}(Y(T)) ;$

$\left.\mathbb{A}_{2}\right)$ для произвольных последовательностей $y_{i} \in W, z_{i} \in F_{i}\left(y_{i}\right)$, обладающих свойствами

$$
\begin{aligned}
\left\|y_{i}\right\|_{W}<R<\infty, \quad & y_{i} \rightarrow y \quad \text { в } \sigma(Y, Z), \quad z_{i} \rightarrow z \quad \text { в } \quad \sigma(Z, Y), \\
& \varlimsup_{i \rightarrow \infty}\left\langle y_{i}, z_{i}\right\rangle \leqslant\langle y, z\rangle,
\end{aligned}
$$

имеет место включение $z \in F_{0}(y)$ и сходимость

$$
\left\langle 1_{[\alpha, t]} y_{i}, z_{i}\right\rangle \rightarrow\left\langle 1_{[\alpha, t]} y, z\right\rangle \text { в } C(T) .
$$


Для стационарной последовательности $F_{i}=F, i=0,1, \ldots$, аналогичные классы м-отображений изучались в [13]-[16]. Из леммы 3 следует, что если последовательность $F_{i}$ удовлетворяет условиям $\left.\left.\mathbb{A}_{1}\right), \mathbb{A}_{2}\right)$, а последовательность $\xi_{i} \rightarrow \xi_{0}$, то и последовательность $F_{i}+\xi_{i}$ удовлетворяет тем же условиям - инвариантность относительно сдвигов. Очевидно, эти условия сохраняются при прореживании последовательности $F_{i}$; если м-отображения $F_{i}$ локальны, то последовательность их сужений на отрезок $J \subset T$ удовлетворяет условиям $\left.\mathbb{A}_{1}\right), \mathbb{A}_{2}$ ) вместе с исходной последовательностью.

Лемма 4. Пусть последовательность м-отображений $F_{i}: Y(T) \rightarrow Z(T)$, $i=0,1, \ldots$, удовлетворяет условиям $\left.\left.\mathbb{A}_{1}\right), \mathbb{A}_{2}\right), h_{i} \in H u h_{i} \rightarrow h_{0}$ в $H$. Пусть $y_{i}, i=1,2, \ldots$, - последовательность решений задач Коши

$$
0 \in y^{\prime}+F_{i}(y), \quad y(\alpha)=h_{i}
$$

и $y_{i} \rightarrow$ в в $\sigma(Y, Z)$. Тогда у есть решение задачи Коши $\left(2_{0}\right)$ и $y_{i} \rightarrow$ у в $C(T, H)$.

ДокАЗАТЕЛЬСТво. Поскольку $-y_{i}^{\prime} \in F_{i}\left(y_{i}\right)$, то найдется такой элемент $z_{i}$ из $F_{i}\left(y_{i}\right)$, что

$$
0=y_{i}^{\prime}+z_{i}, \quad i=1,2, \ldots
$$

Без ограничения обшности можно считать, что $z_{i} \rightarrow z$ в $\sigma(Z, Y)$. Оператор диф्ференцирования слабо замкнут, поэтому $y \in W$ и

$$
0=y+z, \quad y(\alpha)=h_{0} .
$$

Из $\left(2_{i}\right),(6),(7)$ вытекает соотношение

$$
\left\langle 1_{\left[t_{1}, t_{2}\right]}\left(y-y_{i}\right), z_{i}-z\right\rangle=\left.\frac{1}{2}\left|y(t)-y_{i}(t)\right|^{2}\right|_{t_{1}} ^{t_{2}},
$$

$\alpha \leqslant t_{1} \leqslant t_{2} \leqslant \beta, i=1,2, \ldots$ Полагая $t_{1}=\alpha, t_{2}=\beta$, приходим к неравенству (4). Последовательности $y_{i}, z_{i}, i=1,2, \ldots$, обладают свойствами (3), (4). В силу условий $\left.\mathbb{A}_{1}\right), \mathbb{A}_{2}$ ) отсюда следует включение $z \in F_{0}(y)$ и сходимость $(5)$. Так как $z \in F_{0}(y)$, то $y$ есть решение задачи $\left(2_{0}\right)$. Сходимость $y_{i} \rightarrow y$ в $C(T, H)$ вытекает из соотношения (8), в котором $t_{1}=\alpha, t_{2}=t$-произвольное число из $T$. Лемма доказана.

Для локальных м-отображений $F_{i}$ требование $h_{i} \rightarrow h_{0}$ в $H$ можно заменить условием $h_{i} \rightarrow h_{0}$ в $\sigma(H, H)$.

Лемма 5. Пусть последовательность локальных м-отображений $F_{i}$ : $Y(T) \rightarrow Z(T), i=0,1, \ldots$, удовлетворяет условиям $\left.\left.\mathbb{A}_{1}\right), \mathbb{A}_{2}\right), h_{i} \in H \quad u$ $h_{i} \rightarrow h_{0}$ в $\sigma(H, H)$. Пусть $y_{i}, i=1,2, \ldots$, последовательность решений задач Коши $\left(2_{i}\right), y_{i} \rightarrow$ у в $\sigma(Y, Z)$. Тогда у есть решение задачи Коши $\left(2_{0}\right)$ и $(t-\alpha) y_{i} \rightarrow(t-\alpha) y$ в $C(T, H)$.

ДокАЗАТЕЛЬСтво. В силу (6) последовательность $y_{i}$ ограничена в пространстве $W$, а следовательно, компактна в $L^{p}(T, H)$. Производя прореживание и перенумерацию, можно считать, что $y_{i}(t) \rightarrow y(t)$ п.в. в метрике $H$ и $\left(y_{i}(\cdot), v^{*}\right) \rightarrow$ $\left(y(\cdot), v^{*}\right)$ в $C(T)$ для любого $v^{*}$ из $H$. Зафиксируем отрезок $J=[\tau, \beta]$ так, что $\alpha<\tau<\beta$ и $y_{i}(\tau) \rightarrow y(\tau)$ в $H$. Сужение последовательности $F_{i}, i=0,1, \ldots$, на $Y(J)$ удовлетворяет условиям $\left.\left.\mathbb{A}_{1}\right), \mathbb{A}_{2}\right)$. Применяя лемму 4 , получаем, что 
$y_{i} \rightarrow y$ в $C(J, H), y$ есть решение задачи (2) с $F=F_{0}, \alpha=\tau, h=y(\tau)$. В силу произвольности выбора $\tau$ это влечет требуемый результат. Лемма доказана.

Далее м-отображения $F, F_{i}: Y(T) \rightarrow Z(T)$ локальны, $\mathscr{F}\left(\mathscr{F}_{i}\right)$ - порождающие $F\left(F_{i}\right)$ отображения из $T \times V$ в $V^{*}$. Через $U\left(\mathscr{F}_{i}, h_{i}\right)$ обозначим множество решений задачи Коши $\left(2_{i}\right), i=0,1, \ldots$ При определенных предположениях множество $U\left(\mathscr{F}_{i}, h_{i}\right) \subset W$ непусто; его можно рассматривать и как подмножество пространств $Y=Y(T)$ и $X=C(T, H)$. Из лемм 4,5 следует близость последовательности $U\left(\mathscr{F}_{i}, h_{i}\right)$ при $i \gg 1 \mathrm{k} \mathrm{множеству} U\left(\mathscr{F}_{0}, h_{0}\right)$.

ТеОРема 1. Пусть последовательность $F_{i}, i=0,1, \ldots$, удовлетворяет условиям $\left.\left.\mathbb{A}_{1}\right), \mathbb{A}_{2}\right)$. Пусть $U\left(\mathscr{F}_{i}, h_{i}\right) \neq \varnothing, i=1,2, \ldots, u \bigcup_{i} U\left(\mathscr{F}_{i}, h_{i}\right) \subset \mathscr{B}(Y)$. Тогда

1) если $h_{i} \rightarrow h_{0}$ в $H$, mo $U\left(\mathscr{F}_{0}, h_{0}\right) \neq \varnothing u \theta_{X}\left(U\left(\mathscr{F}_{i}, h_{i}\right), U\left(\mathscr{F}_{0}, h_{0}\right)\right) \rightarrow 0$ nрu $i \rightarrow \infty$;

2) если $h_{i} \rightarrow h_{0}$ в $\sigma(H, H)$, mo $U\left(\mathscr{F}_{0}, h_{0}\right) \neq \varnothing u \theta_{X}\left((t-\alpha) U\left(\mathscr{F}_{i}, h_{i}\right)\right.$, $\left.(t-\alpha) U\left(\mathscr{F}_{0}, h_{0}\right)\right) \rightarrow 0$ npu $i \rightarrow \infty$.

ДокаЗАТЕЛЬСТво. Пусть $h_{i} \rightarrow h_{0}$ в $H$. Фиксируем $y_{i}$ из $U\left(\mathscr{F}_{i}, h_{i}\right), i=$ $1,2, \ldots$. Последовательность $y_{i}$ ограничена. Без ограничения общности можно считать, что $y_{i} \rightarrow y$ в $\sigma(Y, Z)$. В силу леммы $4 y \in U\left(\mathscr{F}_{0}, h_{0}\right)$, в частности, $U\left(\mathscr{F}_{0}, h_{0}\right) \neq \varnothing$.

Установим теперь сходимость $\theta_{X}\left(U\left(\mathscr{F}_{i}, h_{i}\right), U\left(\mathscr{F}_{0}, h_{0}\right)\right) \rightarrow 0$ при $i \rightarrow \infty$. Предположим противное. Тогда сушествует такое $\varepsilon>0$, что $\theta_{X}\left(U\left(\mathscr{F}_{i}, h_{i}\right), U\left(\mathscr{F}_{0}, h_{0}\right)\right)>$ $\varepsilon$ для бесконечного числа индексов $i$. Тогда найдется $y_{i}$ из $U\left(\mathscr{F}_{i}, h_{i}\right)$, для которого $\theta_{X}\left(y_{i}, U\left(\mathscr{F}_{0}, h_{0}\right)\right)>\varepsilon$. В силу уже доказанного $\underline{\lim }_{i \rightarrow \infty} \theta_{X}\left(y_{i}, U\left(\mathscr{F}_{0}, h_{0}\right)\right)=0$. Это противоречит неравенству $\theta_{X}\left(y_{i}, U\left(\mathscr{F}_{0}, h_{0}\right)\right)>\varepsilon$.

Второе утверждение теоремы устанавливается на основе леммы 5. Теорема доказана.

Введем еще условие

$\left.\mathbb{A}_{3}\right)$ справедливо неравенство

$$
\left(v, v^{*}\right) \geqslant c_{1}\|v\|^{p}-\gamma_{1}(t)
$$

где $v \in V,|v| \leqslant R, v^{*} \in \mathscr{F}_{i}(t, v), t \in T, i=1,2, \ldots$, постоянная $c_{1}>0$ и функция $\gamma_{1}$ из $L^{1}(T)$ зависят лишь от $R$.

Положим $\mathfrak{B}=\left\{w \in V^{*}:\left\|w ; V^{*}\right\| \leqslant 1\right\}, \mathscr{F}_{i}(t, v ; \rho)=\mathscr{F}_{i}(t, v)+\rho\|v\|^{p-1} \mathfrak{B}$, $\rho \geqslant 0$. Если $v \in V,|v| \leqslant R, \rho \in\left[0, c_{1} / 2\right], v^{*} \in \mathscr{F}_{i}(t, v ; \rho), i=1,2, \ldots$, то из (9) следует оценка

$$
\left(v, v^{*}\right) \geqslant \frac{c_{1}}{2}\|v\|^{p}-\gamma_{1}(t)
$$

Решение $у$ дифференциального включения

$$
0 \in y^{\prime}+\mathscr{F}_{i}(t, y ; \rho)
$$

удовлетворяет вытекающей из (10), (11) оценке

$$
\left(|y(t)|^{2}\right)^{\prime} \leqslant 2 \gamma_{1}(t)-c_{1}\|y(t)\|^{p}
$$


интегрирование которой по отрезку $\left[t_{1}, t_{2}\right], t_{1}<t_{2}$, приводит к неравенству

$$
\left|y\left(t_{2}\right)\right|^{2}-\left|y\left(t_{1}\right)\right|^{2}+c_{1} \int_{t_{1}}^{t_{2}}\|y(t)\|^{p} d t \leqslant 2 \int_{t_{1}}^{t_{2}} \gamma_{1}(t) d t
$$

Из неравенства (12) вытекает, в частности, что ограниченность некоторого множества решений включений (11) в пространстве $X=C(T, H)$ влечет его ограниченность в пространстве $Y(T)$.

ТЕОРЕМа 2. Пусть $T=[0, \omega]$ и выполнень условия $\left.\left.\mathbb{A}_{1}\right)-\mathbb{A}_{3}\right)$. Пусть для всех решений у задачи Коши $\left(2_{0}\right)$ с $\alpha=0,|h| \leqslant R_{0}$ справедливь оченки

$$
|y(t)| \leqslant c_{0}, \quad t \in T, \quad|y(\omega)| \leqslant d_{0},
$$

где $c_{0}>R_{0}, \omega>0, d_{0}>0$. Пусть $\rho_{i} \geqslant 0 u \rho_{i} \rightarrow 0$ при $i \rightarrow \infty$. Тогда для каждого $\varepsilon>0$ можсно указать такое $n_{0}$, что для всех решений у дифференииальных включений (11) с $\rho=\rho_{i}, i>n_{0},|y(0)| \leqslant R_{0}$ выполняются оценки

$$
|y(t)|<c_{0}+\varepsilon, \quad t \in T, \quad|y(\omega)|<d_{0}+\varepsilon
$$

ДокАЗАТЕЛЬСтво. Предположим противное, т.е. что для некоторого $\varepsilon>0$ соответствующее $n_{0}$ не может быть указано. Можно считать, что существует последовательность $y_{i}$ решений включений $(11)$ с $\rho=\rho_{i},\left|y_{i}(0)\right| \leqslant R_{0}$, не удовлетворяющих одному из неравенств (14).

Рассмотрим сначала случай, когда не выполняется первое из неравенств (14). Через $t_{i}$ обозначим наименьшее из чисел $\tau$, для которых $\left|y_{i}(\tau)\right|=c_{0}+\varepsilon$. Таким образом, $\left|y_{i}(t)\right|<c_{0}+\varepsilon, 0 \leqslant t<t_{i},\left|y_{i}\left(t_{i}\right)\right|=c_{0}+\varepsilon$. При $i \gg 1$ для решений $y_{i}(t)$ верны вытекающие из (12) оценки

$$
\left|y_{i}(t)\right|^{2}-\left|y_{i}\left(t_{0}\right)\right|^{2} \leqslant \Gamma_{1}(t)-\Gamma_{1}\left(t_{0}\right)
$$

где $0 \leqslant t_{0}<t \leqslant t_{i}, \Gamma_{1}-$ первообразная функции $2 \gamma_{1}, \Gamma_{1}(0)=0$. Из оценки (15) следует неравенство $\left|y_{i}(t)\right|^{2} \leqslant R_{0}^{2}+\Gamma_{1}(t)$ и отграниченность последовательности $t_{i}$ от 0. Без ограничения обшности можно считать, что $t_{i} \rightarrow t^{*}>0$.

Фиксируем $\beta_{0}$ из $\left(0, t^{*}\right)$ так, что при $i \gg 1$ верно неравенство

$$
\left|y_{i}\left(\beta_{0}\right)\right| \geqslant c_{0}+\frac{\varepsilon}{2}
$$

Возможность подобного выбора следует из (15) и равенства $\left|y_{i}\left(t_{i}\right)\right|=c_{0}+\varepsilon$. Положим $T_{1}=\left[0, \beta_{0}\right]$. Так как $t_{i}>\beta_{0}, i \gg 1$, то $\left\|y_{i} ; C\left(T_{1}, H\right)\right\| \leqslant c_{0}+\varepsilon$. Отсюда в свою очередь вытекает ограниченность последовательности $y_{i}$ в пространстве $Y\left(T_{i}\right)$. Без ограничения общности можно считать, что $y_{i} \rightarrow y$ в $\sigma\left(Y\left(T_{1}\right), Z\left(T_{1}\right)\right)$. В силу леммы $5 y \in U\left(\mathscr{F}_{0}, h_{0}\right)$ с $\left|h_{0}\right| \leqslant R_{0}, t y_{i} \rightarrow t y$ в $C\left(T_{1}, H\right)$. В частности, $\left|y\left(\beta_{0}\right)\right| \leqslant c_{0},\left|y_{i}\left(\beta_{0}\right)\right| \rightarrow\left|y\left(\beta_{0}\right)\right|$ при $i \rightarrow \infty$, что противоречит (16). Полученное противоречие означает, что $\left|y_{i}(t)\right| \leqslant c_{0}+\varepsilon \forall i \gg 1, t \in T=[0, \omega]$.

Повторяя рассуждения предшествуюшего абзаца, можно доказать, что $t y_{i} \rightarrow t y$ в $C(T, H)$; здесь $y$ - решение задачи $\left(2_{0}\right)$ с $\alpha=0,\left|h_{0}\right| \leqslant R_{0}$. Поэтому $\left|y_{i}(\omega)\right| \rightarrow$ $|y(\omega)| \leqslant d_{0}$. В частности, $\left|y_{i}(\omega)\right|<d_{0}+\varepsilon \forall i \gg 1$. Из проведенных рассуждений вытекают оценки (14) при $i \gg 1$. Теорема доказана. 


\section{§ 3. Усреднение дифференциальных включений}

Обсудим условия $\left.\left.\mathbb{A}_{1}\right)-\mathbb{A}_{3}\right)$. Условие $\mathbb{A}_{1}$ ) означает равномерную ограниченность м-отображений $F_{i}: Y \rightarrow Z, i=1,2, \ldots$. Оно может быть выведено из равномерных оценок роста соответствующих м-отображений $\mathscr{F}_{i}: T \times V \rightarrow V^{*}, i=1,2, \ldots$. $\mathrm{C}$ равномерными односторонними оценками роста м-отображений $\mathscr{F}_{i}$ связано неравенство (9), гарантирующее условие $\left.\mathbb{A}_{3}\right)$.

Наименее обозримым представляется условие $\mathbb{A}_{2}$ ). Способы его проверки зависят от выбора последовательности $F_{i}, i=1,2, \ldots$. Остановимся на конструкциях, возникающих при обосновании метода усреднения. В формулируемых ниже предположениях $\left.\left.\mathbb{B}_{1}\right)-\mathbb{B}_{3}\right) Y_{0}$ - всюду плотное в $Y$ пространство, $W_{1}$ - рефлексивное $B$-пространство, $W \stackrel{k}{\longrightarrow} W_{1}, Y \rightarrow W_{1}$. Выбор пространства $W_{1}$ позволяет говорить о полусходящихся последовательностях (см. 1 1).

Сформулируем следующие предположения.

$\left.\mathbb{B}_{1}\right)$ Из соотношений $u \in Y, v \in Y,\|u\|_{Y} \leqslant R,\|v\|_{Y} \leqslant R, u^{*} \in F_{i}(u), v^{*} \in F_{i}(v)$, $i=1,2, \ldots$, следует неравенство

$$
\left\langle u-v, u^{*}-v^{*}\right\rangle \geqslant-c\left(R,\|u-v\|_{W_{1}}\right),
$$

где $c(R, \cdot)$ - неотрицательная непрерьвная функция, не зависящая от $i$; $c(R, \xi)=o(\xi)$ при $\xi \rightarrow+0$.

$\left.\mathbb{B}_{2}\right)$ Если $u \in Y_{0}$, то для каждого бесконечного множества $\mathbb{N}_{0}$ натуральных чисел существует полусходяшаяся подпоследовательность $\xi_{i} \in F_{i}(u), i \in$ $\mathbb{N}_{0}$.

$\mathbb{B}_{3}$ ) Отображение $F_{0}: Y \rightarrow \operatorname{Cv}(Z)$ - ограниченное хеминепрерывное сверху м-отображение и для любых $u \in Y_{0}, w \in Y$

$$
\varlimsup_{i \rightarrow \infty} s\left(w, F_{i}(u)\right) \leqslant s\left(w, F_{0}(u)\right)
$$

Лемма 6. Пусть последовательность м-отображсений $F_{i}: Y \rightarrow Z, i=$ $0,1, \ldots$, удовлетворяет условию $\left.\mathbb{A}_{1}\right)$ и предполодсениям $\left.\left.\mathbb{B}_{1}\right)-\mathbb{B}_{3}\right)$. Тогда вьполнено условие $\mathbb{A}_{2}$ ).

ДокАЗАТЕльство. Зафиксируем $u$ из $Y_{0}$, последовательности $y_{i} \in W, z_{i} \in$ $F_{i}\left(y_{i}\right), i=1,2, \ldots$, и элементы $y, z$, обдающие свойствами $(3),(4)$. Не уменьшая обшности, можно считать, что $y_{i} \rightarrow y$ в $W_{1}$ и имеется последовательность $\xi_{i} \in$ $F_{i}\left(u_{i}\right), \xi_{i} \rightarrow \xi$ и $\xi_{i} \rightarrow \xi$ в $\sigma(Z, Y)$. Так как $z_{i} \in F_{i}\left(y_{i}\right), \xi_{i} \in F_{i}(u)$, то в силу (17)

$$
\left\langle y_{i}-u, z_{i}-\xi_{i}\right\rangle \geqslant-c\left(R,\left\|y_{i}-u\right\|_{W_{1}}\right),
$$

$R>\left\|y_{i}\right\|_{Y}, R>\|y\|_{Y}$. Используя неравенство (4) и лемму 3, отсюда получаем $\langle y-u, z-\xi\rangle \geqslant-c\left(R,\|y-u\|_{W_{1}}\right)$. Так как

$$
\langle u-y, \xi\rangle=\lim _{i \rightarrow \infty}\left\langle u-y, \xi_{i}\right\rangle \leqslant \varlimsup_{i \rightarrow \infty} s\left(u-y, F_{i}(u)\right) \leqslant s\left(u-y, F_{0}(u)\right),
$$

TO

$$
s\left(u-y, F_{0}(u)\right) \geqslant\langle u-y, z\rangle-c\left(R,\|y-u\|_{W_{1}}\right) .
$$

В этом неравенстве $u$ - произвольньй элемент из $Y_{0}$. Используя хеминепрерывность сверху м-отображения $F_{0}: Y \rightarrow \mathrm{Cv}(Z)$ и плотность $Y_{0}$ в $Y$, распространяем 
неравенство (20) на все функции $u$ из $Y$. Полагая в (20) $u=y+\lambda v, v \in Y, \lambda>0$, приходим (как и при доказательстве леммы 1$)$ к оценке $s\left(v, F_{0}(y)\right) \geqslant\langle v, z\rangle$. В силу произвольности $v$ из $Y$ отсюда следует включение $z \in F_{0}(y)$.

Неравенство (19) приводит к соотношению

$$
\begin{aligned}
\varliminf_{i \rightarrow \infty}\left\langle y_{i}, z_{i}\right\rangle & \geqslant \varliminf_{i \rightarrow \infty}\left(\left\langle u, z_{i}-\xi_{i}\right\rangle+\left\langle y_{i}, \xi_{i}\right\rangle-c\left(R,\left\|y_{i}-u\right\|_{W_{1}}\right)\right) \\
& \geqslant\langle y-u, \xi\rangle+\langle u, z\rangle-c\left(R,\|y-u\|_{W_{1}}\right)
\end{aligned}
$$

из которого получаем неравенство

$$
\lim _{i \rightarrow \infty}\left\langle y_{i}, z_{i}\right\rangle \geqslant\langle y, z\rangle
$$

Предположим теперь, что $0 \in \mathscr{F}_{i}(t, 0), i=1,2, \ldots$. Это дополнительное предположение гарантирует следующее свойство м-отображений $F_{i}$ : если $z \in F_{i}(y), \Delta$ - измеримое подмножество отрезка $T$, то $1_{\Delta} z \in F_{i}\left(1_{\Delta} y\right)$. Данное свойство позволяет заменить в неравенстве (19) функции $y_{i}, u, z_{i}, \xi_{i}$ функциями $1_{\Delta} y_{i}, 1_{\Delta} u, 1_{\Delta} z_{i}, 1_{\Delta} \xi_{i}$. Это приводит к оценке $\underline{\lim }_{i \rightarrow \infty}\left\langle 1_{\Delta} y_{i}, 1_{\Delta} z_{i}\right\rangle \geqslant\left\langle 1_{\Delta} y, 1_{\Delta} z\right\rangle$. Последняя оценка имеет место и при замене множества $\Delta \subset T$ на его дополнение $T \backslash \Delta$. Вместе с неравенством (4) это влечет сходимость $\left\langle 1_{\Delta} y_{i}, 1_{\Delta} z_{i}\right\rangle \rightarrow\left\langle 1_{\Delta} y, 1_{\Delta} z\right\rangle$. Следовательно, $\left(y_{i}, z_{i}\right) \rightarrow(y, z)$ в $\sigma\left(L^{1}, L^{\infty}\right)$. Теперь вьполнение условия $\left.\mathbb{A}_{2}\right)$ очевидно.

От предположения $0 \in \mathscr{F}_{i}(t, 0)$ можно избавиться с помощью подходящих сдвигов. Действительно, не уменьшая обшности, будем считать, что сушествует полусходяшаяся последовательность $\stackrel{\circ}{\xi}_{i} \in F_{i}(0)$. Тогда приведенное выше рассуждение применимо к последовательности $F_{i}-\stackrel{\circ}{\xi}_{i}$. Следовательно, исходная последовательность $F_{i}$ удовлетворяет условию $\left.\mathbb{A}_{2}\right)$. Лемма доказана.

Предположение $\mathbb{B}_{1}$ ) близко к условию равномерной полуограниченности вариаций м-отображений $F_{i}: Y \rightarrow \operatorname{Cv}(Z), i=1,2, \ldots$ (см. [9]); $\mathbb{B}_{2}$ ) имеет характер условия компактности последовательности $F_{i}(u)$ (это особенно явно для однозначных отображений $\left.\left.F_{i}\right) ; \mathbb{B}_{3}\right)$ связывает последовательность $F_{i}$ с предельньм отображением $F_{0}$. Неравенство (18) приводит лишь к оценкам снизу размеров множества $F_{0}(u)$.

Аналогичные (18) односторонние оценки характерны для рассматриваемой ниже модификации метода усреднения. Далее $\mathbb{R}_{+}=[0, \infty), E_{0}$-плотное в пространстве $V$ подпространство. М-отображения $\mathscr{F}: \mathbb{R}_{+} \times V \rightarrow V^{*}$ и $\mathscr{F}_{0}: V \rightarrow \operatorname{Cv}\left(V^{*}\right)$ назовем допустимой парой, если

1) сужение $\mathscr{F}$ на множества вида $[\alpha, \beta] \times V$ удовлетворяет слабому условию Каратеодори;

2) $\mathscr{F}_{0}: V \rightarrow \mathrm{Cv}\left(V^{*}\right)$ - ограниченное хеминепрерьвное сверху м-отображение;

3) для любых $v, w$ из $E_{0}, t_{0} \in \mathbb{R}_{+}$имеет место неравенство

$$
\varlimsup_{N \rightarrow \infty} \frac{1}{N} \int_{t_{0}}^{t_{0}+N} s(w, \mathscr{F}(t, v)) d t \leqslant s\left(w, \mathscr{F}_{0}(v)\right)
$$


Если $\mathscr{F}$ - сужение на $\mathbb{R}_{+} \times V \omega$-периодического по $t$ отображения $\mathscr{F}: \mathbb{R} \times V \rightarrow$ $\mathrm{Cv}\left(V^{*}\right)$, то отображение $\mathscr{F}_{0}$ можно определить равенством

$$
\mathscr{F}_{0}(v)=\frac{1}{\omega} \int_{0}^{\omega} \mathscr{F}(t, v) d t,
$$

интеграл м-отображения $t \mapsto \mathscr{F}(t, v)$ определяется стандартньм образом [2]-[8]. В этом случае верхний предел в (21) можно заменить обычным пределом, неравенство (21) становится равенством. В обшем случае связь между м-отображениями $\mathscr{F}$ и $\mathscr{F}_{0}$ односторонняя; не исключается, например, ситуация, ког да $\mathscr{F}$ однозначно, а $\mathscr{F}_{0}$ многозначно.

Сопоставим допустимой паре $\mathscr{F}, \mathscr{F} 0$ семейство м-отображений $\mathscr{F}(\cdot, \cdot ; \lambda, \tau)$ : $\mathbb{R}_{+} \times V \rightarrow V^{*}, \lambda \geqslant 1, \tau \geqslant 0$, определяемых равенствами

$$
\mathscr{F}(t, v ; \lambda, \tau)= \begin{cases}\mathscr{F}(\lambda t+\tau, v), & \text { если } 1 \leqslant \lambda<\infty ; \\ \mathscr{F}_{0}(v), & \text { если } \lambda=\infty .\end{cases}
$$

Ниже $\Pi\left(T, E_{0}\right)$ - линейное подпространство $Y(T)$, состоящее из функций $y: T \rightarrow E_{0}$, постоянных на промежутках, образующих конечное разбиение отрезка $T \subset \mathbb{R}_{+}$. Несложно доказать плотность $\Pi\left(T, E_{0}\right)$ в пространстве $Y(T)$. Сужение $\mathscr{F}(\cdot, \cdot ; \lambda, \tau)$ на $T \times V$ принадлежит классу $\mathbb{C}_{*}\left(T, V, V^{*}\right)$, поэтому оно порождает оператор суперпозиции $F(\cdot ; \lambda, \tau)$. Если $y \in L^{\infty}(T, V)$, то $F(y ; \lambda, \tau) \in \operatorname{Cv}\left(L^{1}\left(T, V^{*}\right)\right)$. Из $(21)$ вытекает неравенство

$$
\varlimsup_{\lambda \rightarrow \infty} s(w, F(u ; \lambda, \tau)) \leqslant s(w, F(u ; \infty, \tau)),
$$

в котором $w, u \in \Pi\left(T, E_{0}\right), \tau \geqslant 0$.

При дополнительных предположениях о росте м-отображений $\mathscr{F}, \mathscr{F} 0$ операторы суперпозиции $F(\cdot ; \lambda, \tau)$ действуют и ограничены из $Y=Y(T)$ в $Z=Z(T)$. Дифференциальное включение $0 \in y^{\prime}+F(y ; \lambda, \tau)$ записьваем далее в виде

$$
\begin{array}{lr}
0 \in y^{\prime}+\mathscr{F}(\lambda t+\tau, y), & 1 \leqslant \lambda<\infty, \\
0 \in y^{\prime}+\mathscr{F}_{0}(y), & \lambda=\infty,
\end{array}
$$

соответственно. Через $\mathscr{N}(\lambda, \tau ; h)\left(\mathscr{N}_{0}(h)\right)$ обозначаем множество решений включения (23) (соответственно (24)), удовлетворяющих начальному условию $y(0)=$ $h$. Включение (24) естественно назвать усреднением включения (23); описание других версий метода усреднения можно найти, например, в [2], [3], [10], [17]-[19].

Из теорем 1, 2 может быть выведена близость множества $\mathcal{N}(\lambda, \tau ; h)$ к множеству $\mathscr{N}_{0}(h)$. Ниже $T=[0, \omega], \omega-$ произвольное положительное число, $F(\cdot ; \lambda, \tau)$, $\lambda \geqslant 1, \tau \geqslant 0,-$ оператор суперпозиции, порождаемый м-отображением $\mathscr{F}: \mathbb{R} \times$ $V \rightarrow V^{*}$. Через $M^{r}(\mathbb{R}, E)$ обозначается пространство ограниченных по Степанову функций на $\mathbb{R}_{+}$со значениями в $B$-пространстве $E$. Оно состоит из измеримых по Лебегу-Бохнеру функций $f: \mathbb{R}_{+} \rightarrow E$, для которых имеет смысл и конечна норма $\left\|f ; M^{r}\right\|=\sup \left\{\left\|f ; L^{r}(I, E)\right\|\right\}$, где sup берется по всем отрезкам $I$ единичной длины. Полагаем $M^{r}\left(\mathbb{R}_{+}\right)=M^{r}\left(\mathbb{R}_{+}, \mathbb{R}\right)$. Введем условия $\left.\left.\mathbb{C}_{1}\right)-\mathbb{C}_{3}\right)$ :

$\left.\mathbb{C}_{1}\right) F\left(M \times[1, \infty) \times \mathbb{R}_{+}\right) \in \mathscr{B}(Z(T)) \forall M \in \mathscr{B}(Y(T)) ;$

$\mathbb{C}_{2}$ ) для произвольной последовательности $\left(\lambda_{i}, \tau_{i}\right), \lambda_{i} \geqslant 1, \tau_{i} \geqslant 0, \lambda_{i} \rightarrow \infty$, соответствуюшая последовательность операторов суперпозиции $F_{i}=$ $F\left(\cdot ; \lambda_{i}, \tau_{i}\right)$ удовлетворяет условию $\left.\mathbb{A}_{2}\right) ;$

$\mathbb{C}_{3}$ ) справедливы неравенства (9), где $v \in V,|v| \leqslant R, t \in \mathbb{R}_{+}, v^{*} \in \mathscr{F}(t, v)$, постоянная $c_{1}>0$ и функция $\gamma_{1}$ из $M^{1}\left(\mathbb{R}_{+}\right)$зависят лишш от $R$. 
Условие $\mathbb{C}_{j}$ ) влечет выполнение условия $\mathbb{A}_{j}$ ) для каждой последовательности $F_{i}=F\left(\cdot ; \lambda_{i}, \tau_{i}\right), \lambda_{i} \geqslant 1, \tau_{i} \geqslant 0, \lambda_{i} \rightarrow \infty$. В соответствии с теоремой 1 это позволяет гарантировать близость множества $\mathscr{N}(\lambda, \tau ; h), \lambda \gg 1$, к множеству $\mathscr{N}_{0}(h)$ в метрике пространства $X=C(T, H)$.

ТеОрема 3. Пусть выполнены условия $\left.\left.\mathbb{C}_{1}\right), \mathbb{C}_{2}\right)$. Пусть $\mathscr{N}(\lambda, \tau ; h) \neq \varnothing$, $\lambda \geqslant \lambda_{0} \gg 1, u \bigcup_{\lambda \geqslant \lambda_{0}, \tau \geqslant 0} \mathscr{N}(\lambda, \tau ; h) \subset \mathscr{B}(Y)$. Тогдa $\mathscr{N}_{0}(h) \neq \varnothing u$

$$
\theta_{X}\left(\mathscr{N}(\lambda, \tau ; h), \mathscr{N}_{0}(h)\right) \rightarrow 0, \quad \lambda \rightarrow \infty
$$

равномерно по $\tau \geqslant 0$.

ДоказАТЕЛЬство. Теорема 3 есть следствие теоремы 1.

Наиболее затруднительна проверка условия $\mathbb{C}_{2}$ ); она может основываться на непрерывных аналогах предположений $\left.\left.\mathbb{B}_{1}\right)-\mathbb{B}_{3}\right)$. Если отображение $\mathscr{F}(t, \cdot): V \rightarrow$ $V^{*}$ монотонно, то имеет место неравенство (17) с $c(R, \cdot)=0$. Монотонность м-отображения $\mathscr{F}(t, \cdot)$ можно заменить менее ограниченными условиями (см. [9], [14]). Для проверки предположения $\left.\mathbb{B}_{3}\right)$ полезны лемма 1 и неравенство (22).

Перейдем к предположению $\left.\mathbb{B}_{2}\right)$, считая, что $Y_{0}=\Pi\left(T, E_{0}\right), W_{1}=L^{p}(T, E) \cap$ $L^{r}(T, H)$, где $E$ - реффлексивное пространство, $V \stackrel{k}{\longrightarrow} E \rightarrow H, 1<r<\infty$; в этом случае справедливы вложения $W \stackrel{k}{\longrightarrow} W_{1}, Y \rightarrow W_{1}$ (см. $\left.\S 1\right)$.

ЛЕмма 7. Пусть для любого $v_{0}$ из $E_{0}$ существует сечение м-отображения $t \mapsto \mathscr{F}\left(t, v_{0}\right)$ вида $v_{0}^{*}+z_{1}+z_{2}$, где $v_{0}^{*} \in V^{*}, z_{1} \in M^{q}\left(\mathbb{R}_{+}, E^{*}\right), z_{2} \in M^{s}\left(\mathbb{R}_{+}, H\right)$, $1 / r+1 / s=1$. Тогда для всякой последовательности м-отображений $F_{i}=$ $F\left(\cdot ; \lambda_{i}, \tau_{i}\right): Y(T) \rightarrow Z(T), \lambda_{i} \rightarrow \infty, \tau_{i} \geqslant 0$, вьполнено предположение $\left.\mathbb{B}_{2}\right)$.

ДоказАтЕльство. Множество функций вида $t \mapsto z_{1}(\lambda t+\tau), t \in T, \lambda \geqslant 1$, $\tau \geqslant 0$, ограничено в пространстве $L^{q}\left(T, E^{*}\right)$, а множество функций $t \mapsto z_{2}(\lambda t+\tau)$ - в пространстве $L^{s}(T, H)$. Отсюда без труда выводится требуемое утверждение. Лемма доказана.

Приведем теперь вариант теоремы 2 , относящийся к дифференциальному включению с параметрами $\lambda \geqslant 1, \tau \geqslant 0, \rho \geqslant 0$ :

$$
0 \in y^{\prime}+\mathscr{F}(\lambda t+\tau, y)+\rho\|y\|^{p-1} \mathfrak{B}
$$

как и в $\S 2, \mathfrak{B}=\left\{w \in V^{*}:\left\|w ; V^{*}\right\| \leqslant 1\right\}$. Включение (25) можно рассматривать как возмущение включения (23).

Теорема 4. Пусть выполнены условия $\left.\left.\mathbb{C}_{1}\right)-\mathbb{C}_{3}\right)$. Пусть для всех у из $\mathscr{N}_{0}(h)$, $|h| \leqslant R_{0}$, справедливы оценки (13) с $T=[0, \omega], c_{0}>R_{0}, \omega>0, \delta>0$. Тогда для каждого $\varepsilon>0$ можно указать такие числа $\lambda_{0}>1, \delta>0$, что для всех решений у включения (25) $с \lambda>\lambda_{0}, 0 \leqslant \rho<\delta, \tau \geqslant 0,|y(0)| \leqslant R_{0}$ имеют место оценки (14).

Теорема 4 есть переформулировка теоремы 2 на язьке $\varepsilon-\delta$, поэтому ее доказательство не приводится. 


\section{§4. Асимптотика решений дифференциальных включений}

Далее дифференциальные включения (23)-(25) изучаются при дополнительном предположении $(p-1)$-однородности отображений $\mathscr{F}(t, v), \mathscr{F} 0(v)$ по фазовому переменному $v$ :

$$
\mathscr{F}(t, k v)=k^{p-1} \mathscr{F}(t, v), \quad \mathscr{F}_{0}(k v)=k^{p-1} \mathscr{F}_{0}(v)
$$

$t \in \mathbb{R}_{+}, v \in V, k>0$. Через $\mathfrak{N}(\lambda, \tau, \rho ; h)$ обозначается множество решений $y(t)$, $t \geqslant 0$, включения (25), удовлетворяющих начальному условию $y(0)=h$. Из $(p-1)$-однородности отображения $\mathscr{F}$ вытекает следующее свойство инвариантности класса $\mathfrak{N}(\lambda, \tau, \rho ; h)$ : если $y \in \mathfrak{N}(\lambda, \tau, \rho ; h)$, то при любых $R>0, t_{0} \geqslant 0$ функция

$$
y_{1}(t)=\frac{y\left(t_{0}+R^{2-p} t\right)}{R}
$$

принадлежит классу $\mathfrak{N}\left(\lambda_{1}, \tau_{1}, \rho ; h_{1}\right)$ с $\lambda_{1}=R^{2-p} \lambda, \tau_{1}=t_{0}+R^{2-p} \tau, h_{1}=y\left(t_{0}\right) / R$. Очевидно равенство $\mathscr{N}(\lambda, \tau ; h)=\mathfrak{N}(\lambda, \tau, 0 ; h)$.

Так как $0 \in \mathscr{F}(t, 0), t \in \mathbb{R}$, то функция $y_{0}(t) \equiv 0$ есть решение включения (25) при любых параметрах $\lambda, \tau, \rho$. Назовем нулевое решение включения (25) асимптотически устойчивым, если

1) для каждого $\varepsilon>0$ найдется такое $\eta>0$, что любая функция $y$ из $\mathfrak{N}(\lambda, \tau, \rho ; h),|h|<\eta$, удовлетворяет неравенству $|y(t)|<\varepsilon ;$

2) $|y(t)| \rightarrow 0$ при $t \rightarrow \infty$.

Аналогичньм образом определяется асимптотическая устойчивость нулевого решения включения (24). Как и в $\S 3, \mathscr{N}_{0}(h)$ - множество решений включения $(24)$, удовлетворяющих начальному условию $y(0)=h$. Будем писать $\mathscr{F}_{0} \in \mathfrak{B}_{p}(V)$, если все функции из $\mathscr{N}_{0}(h),|h| \leqslant 1$, равномерно ограничены и равномерно стремятся к нулю (в метрике $H$ ) при $t \rightarrow \infty$. Включение $\mathscr{F}_{0} \in \mathfrak{B}_{p}(V)$ влечет асимптотическую устойчивость нулевого решения включения (24); при некоторых дополнительных предположениях верно и обратное [7], [20].

ТеОРема 5. Пусть допустимая пара м-отображений $\mathscr{F}: \mathbb{R}_{+} \times V \rightarrow V^{*}$, $\mathscr{F}_{0}: V \rightarrow \operatorname{Cv}\left(V^{*}\right)$ удовлетворяет условиям $\left.\left.\mathbb{C}_{1}\right)-\mathbb{C}_{3}\right)$ и условию однородносmu (26). Пусть $\mathscr{F}_{0} \in \mathfrak{B}_{p}(V)$.

Тогда найдутся такие полохсительнье постоянные $k_{1}, k_{2}, k, \delta_{0}, r_{0}, \lambda_{0}$, чmo

1) если $p>2$, то для у из $\mathfrak{N}(1, \tau, \rho ; h), \tau \in \mathbb{R}_{+}, 0 \leqslant \rho<\delta_{0},|h|<r_{0}$, верна оченка

$$
|y(t)| \leqslant \min \left\{k_{1}|y(0)|, k_{2} t^{1 /(2-p)}\right\}, \quad t \geqslant 0
$$

2) если $p=2$, то для у из $\mathfrak{N}(\lambda, \tau, \rho ; h), \tau \geqslant 0, \lambda>\lambda_{0}, 0 \leqslant \rho<\delta_{0}$, справедлива оченка

$$
|y(t)| \leqslant k_{1}|y(0)| e^{-k t}, \quad t \geqslant 0
$$


ДокаЗАТЕЛЬСтво. Шаг $1: p \geqslant 2$. Так как $\mathscr{F}_{0} \in \mathfrak{B}_{p}(V)$, то найдутся такие постоянные $c_{0}>0, c_{1} \in\left(0, \frac{1}{2}\right), \omega>0$, что для всех решений $y$ из $\mathscr{N}_{0}(h),|h| \leqslant 1$, верны оценки

$$
|y(t)| \leqslant c_{0}, \quad 0 \leqslant t \leqslant \omega, \quad|y(\omega)| \leqslant c_{1} .
$$

Фиксируем $\varepsilon>0$ так, что $c_{1}+\varepsilon<\frac{1}{2}$. Согласно теореме 4 найдутся такие числа $\lambda_{0}>1, \delta_{0}>0$, что для всех решений $u$ включения (25) с $\lambda>\lambda_{0}, 0 \leqslant \rho<\delta_{0}, \tau \geqslant 0$, $|u(0)| \leqslant 1$ имеет место оценка

$$
|u(t)|<c_{0}+\varepsilon, \quad 0 \leqslant t \leqslant \omega, \quad|u(\omega)|<c_{1}+\varepsilon<\frac{1}{2} .
$$

$\mathrm{B}$ частности, если $|u(0)|=1$, то существует такое число $t^{*} \in(0, \omega)$, что $\frac{1}{2}<$ $|u(t)|<c_{0}+\varepsilon, 0 \leqslant t<t^{*},\left|u\left(t^{*}\right)\right|=\frac{1}{2}$; число $t^{*}$ может зависеть от $u$.

Пусть $v \in \mathfrak{N}(\lambda, \tau, \rho ; h)$ с $\lambda>\lambda_{0}, \tau \geqslant 0,0 \leqslant \rho<\delta_{0},|h|=1$. Положим $t_{0}=0, R_{i}=2^{-i}$. Установим сушествование последовательности $t_{i}, i=1,2, \ldots$, обладаюшей свойствами

$$
|v(t)| \leqslant 2\left(c_{0}+\varepsilon\right) R_{i}, \quad t_{i-1} \leqslant t \leqslant t_{i}, \quad\left|v\left(t_{i}\right)\right|=R_{i}, \quad 0<t_{i+1}-t_{i}<\omega R_{i}^{2-p} .
$$

Сушествование $t_{1}$ установлено выше. Если известно $t_{i}$, то для построения $t_{i+1}$ введем функцию

$$
u(t)=\frac{v\left(R_{i}^{2-p} t+t_{i}\right)}{R_{i}} .
$$

В силу свойства инвариантности $u \in \mathfrak{N}\left(\lambda_{i}, \tau_{i}, \rho ; h_{i}\right)$ с $\lambda_{i}=\lambda R_{i}^{2-p} \geqslant \lambda_{0}, \tau_{i} \geqslant 0$, $0 \leqslant \rho<\delta_{0},\left|h_{i}\right|=1$, поэтому $\frac{1}{2}<|u(t)|<c_{0}+\varepsilon, 0 \leqslant t<t^{*},\left|u\left(t^{*}\right)\right|=\frac{1}{2}, t^{*}-$ некоторое число из $(0, \omega)$. Теперь достаточно положить $t_{i+1}=t_{i}+t^{*} R_{i}^{2-p}$.

Шаг 2: $p>2$. Из неравенства (27) следует, что функция $v$ из $\mathfrak{N}(\lambda, \tau, \rho ; h)$, $\lambda>\lambda_{0}, \tau \geqslant 0,0 \leqslant \rho<\delta_{0},|h|=1$, удовлетворяет оценке

$$
|v(t)| \leqslant \frac{c_{0}+\varepsilon}{2^{i-1}}, \quad t \geqslant k_{0} 2^{i(p-2)},
$$

где $k_{0}$ - постоянная, не зависящая от $v$ и $i$. Непосредственным следствием этой оценки является неравенство

$$
|v(t)| \leqslant \min \left\{\varkappa_{1}, \varkappa_{2} t^{1 /(2-p)}\right\},
$$

постоянные $\varkappa_{1}>0, \varkappa_{2}>0$ не зависят от $v$.

Оценка (28) влечет первое утверждение теоремы. Действительно, пусть $r_{0}>0$ и $r_{0}^{2-p}>\lambda_{0}$. Если $y \in \mathfrak{N}(1, \tau, \rho ; h), \tau \geqslant 0,0 \leqslant \rho<\delta_{0}, 0<|h|<r_{0}$, то в силу свойства инвариантности функция $v(t)=y\left(|h|^{2-p} t\right) /|h|$ принадлежит $\mathfrak{N}\left(\lambda_{1}, \tau_{1}, \rho ; h_{1}\right)$ с $\lambda_{1}=|h|^{2-p}>\lambda_{0}$, поэтому для нее верна оценка (28). Следовательно,

$$
\left|y\left(|h|^{2-p} t\right)\right| \leqslant|h| \min \left\{\varkappa_{1}, \varkappa_{2} t^{1 /(2-p)}\right\} .
$$

Отсюда вытекает требуемое утверждение.

Шаг 3: $p=2$. Оценка (27) сохраняется при $p=2$. Она приводит к оценке

$$
|v(t)| \leqslant \frac{c_{0}+\varepsilon}{2^{i-1}}, \quad t \geqslant k_{0} i
$$


где $k_{0}$ - постоянная, не зависящая от $i$ и функции $v$ из $\mathfrak{N}(\lambda, \tau, \rho ; h), \lambda>\lambda_{0}, \tau \geqslant 0$, $0 \leqslant \rho<\delta_{0},|h|=1$. Ее следствием является неравенство $|v(t)| \leqslant \varkappa \exp (-k t)$, постоянные $\varkappa>0, k>0$ не зависят от $v$. Теперь второе утверждение выводится из 1-однородности $\mathscr{F}$. Теорема доказана.

В предположениях теоремы 5 нулевое решение включения (25) асимптотически устойчиво, если $0 \leqslant \rho<\delta_{0}$. Отсюда обычным образом (см., например, [7], [20]) вьводится асимптотическая устойчивость нулевого решения дифференциального включения с однородной главной частью.

Отображение $\mathscr{F}_{0}: V \rightarrow V^{*}$ назовем положительно определенным, если $\left(v, v^{*}\right) \geqslant \varkappa_{0}|v|^{p}$, где $v \in V, v^{*} \in \mathscr{F}_{0}(v), \varkappa_{0}$ - положительная постоянная. Положительная определенность $\mathscr{F}_{0}$ гарантирует включение $\mathscr{F}_{0} \in \mathfrak{B}_{p}(V)$. Для потенциальных операторов известны частичные обрашения этого результата [20], [21].

В заключение рассмотрим пример иллюстративного характера. Пусть $\Omega$ - ограниченная область в $\mathbb{R}^{n}, \stackrel{\circ}{W}{ }_{p}^{1}(\Omega)$ - пространство Соболева [22; гл. 1]. Пространство $V=\stackrel{\circ}{W} \underset{p}{1}(\Omega)$ с нормой $\|u\|=\left\|\nabla u ; L^{p}\right\|$ компактно вложено в пространство $H=L^{2}(\Omega)$ с нормой $|u|=\left\|u ; L^{2}\right\|$. Пара пространств $V, H$ и числа $p, p_{0}$, $2 \leqslant p \leqslant p_{0}<\infty$, однозначно определяют соответствуюшие им пространства $Y, Z, W$ функций на отрезке $T \subset \mathbb{R}$. В частности, $Y=L^{p}(T, \stackrel{\circ}{W} \underset{p}{1}(\Omega)) \cap$ $L^{\infty}\left(T, L^{2}(\Omega)\right) ; Y$ можно интерпретировать как пространство функций на цилиндpe $Q=T \times \Omega$. Фиксируем $r_{1}$ из $(p, p(1+2 / n))$. Будем считать $p_{0}$ настолько большим, что $Y \rightarrow L^{r_{1}}(Q)$. Это предположение гарантирует вложение $L^{s_{1}}(Q) \rightarrow Z$, $1 / r_{1}+1 / s_{1}=1$.

Пусть $a(t, x), b(t, x), t \in \mathbb{R}, x \in \Omega,-\omega$-периодические по $t$ функции, сужения которых на цилиндр $Q=T \times \Omega, T=[0, \omega], 0<\omega<\infty$, принадлежат пространству $L^{\infty}(Q)$. Будем считать, что $a(t, x) \geqslant \varepsilon_{0}>0$ и $a(t, x)=a_{1}(t, x)+a_{2}(x)$, где $a_{1}$ и $\partial a_{1} / \partial x_{j}, j=1, \ldots, n,-$ функции класса $L^{\infty}(Q)$. Положим

$$
a_{0}(x)=\frac{1}{\omega} \int_{0}^{\omega} a(t, x) d t, \quad b_{0}(x)=\frac{1}{\omega} \int_{0}^{\omega} b(t, x) d t .
$$

Введем отображения $\mathscr{F}: \mathbb{R}_{+} \times V \rightarrow V^{*}, \mathscr{F}_{0}: V \rightarrow V^{*}$ соотношениями

$$
\begin{aligned}
\mathscr{F}(t, v) & =-\operatorname{div}\left(a(t, \cdot)|\nabla v|^{p-2} \nabla v\right)+b(t, \cdot)|v|^{p-2} v, \\
\mathscr{F}_{0}(v) & =-\operatorname{div}\left(a_{0}(\cdot)|\nabla v|^{p-2} \nabla v\right)+b(\cdot)|v|^{p-2} v .
\end{aligned}
$$

В частности, $v^{*}=\mathscr{F}(t, v)$ - это функционал на пространстве $V=\stackrel{\circ}{{ }^{\prime}} p(\Omega)$, определяемый равенством

$$
\left(w, v^{*}\right)=\int_{\Omega}\left(\nabla w \cdot a(t, \cdot)|\nabla v|^{p-2} \nabla v+w b(t, \cdot)|v|^{p-2} v\right) d x, \quad w \in V .
$$

Отображения $\mathscr{F}(t, v), \mathscr{F}_{0}(v), t \in \mathbb{R}_{+}, v \in V$, образуют допустимую пару и $(p-1)$-однородны по $v$. Проверим условия $\left.\left.\mathbb{C}_{1}\right)-\mathbb{C}_{3}\right)$. Условие $\left.\mathbb{C}_{1}\right)$ вытекает из включений $a \in L^{\infty}(Q), b \in L^{\infty}(Q)$. Отображение $\mathscr{F}_{0}: V \rightarrow V^{*}$ ограничено и непрерывно. Неравенство $a(t, x) \geqslant \varepsilon_{0}>0$ и ограниченность функции $b(\cdot)$ влекут оценку

$$
(v, \mathscr{F}(t, v)) \geqslant \varkappa_{1}\|v\|^{p}-\varkappa_{2}\left\|v ; L^{p}\right\|^{p}
$$


постоянные $\varkappa_{1}>0, \varkappa_{2}>0$ не зависят от $v$ из $V$. Объединяя эту оценку с неравенством $\left\|v ; L^{p}\right\| \leqslant \delta\|v\|+c(\delta)|v|$, где $v \in V, \delta$ - произвольная положительная постоянная, константа $c(\delta)$ не зависит от $v$, приходим к неравенству

$$
(v, \mathscr{F}(t, v)) \geqslant \varkappa_{3}\|v\|^{p}-\varkappa_{4}|v|^{p},
$$

$\varkappa_{3}>0, \varkappa_{4}>0, v \in V$, из которого следует выполнимость условия $\left.\mathbb{C}_{3}\right)$.

Проверка условия $\mathbb{C}_{2}$ ) основана на лемме 6 , относящейся к случаю $W_{1}=L^{r_{1}}(Q)$. Определяемый равенством $G_{0}(y)=|y|^{p-2} y$ оператор суперпозиции действует и непрерывен из $L^{r_{1}}(Q)$ в $L^{s_{1}}(Q)$; на каждом шаре пространства $L^{r_{1}}(Q)$ он равномерно непрерывен $[12 ;$ гл. 5]. В частности, справедлива оценка

$$
\left\|G_{0}\left(y_{1}\right)-G_{0}\left(y_{2}\right) ; L^{s_{1}}\right\| \leqslant \psi_{\rho}\left(\left\|y_{1}-y_{2} ; L^{r_{1}}\right\|\right) ;
$$

здесь $\left\|y_{j} ; L^{r_{1}}\right\| \leqslant \rho, j=1,2, \psi_{\rho}(+0)=0$. Оператор $G(y)=b(\cdot) G_{0}(y)$ действует и непрерывен из $Y$ в $Z$. Справедлива вытекающая из (29) оценка

$$
\left\langle G\left(y_{1}\right)-G\left(y_{2}\right), y_{1}-y_{2}\right\rangle \geqslant-c\left(R,\left\|y_{1}-y_{2} ; L^{r_{1}}\right\|\right)
$$

где $\left\|y_{j} ; Y\right\| \leqslant R, j=1,2$, функция $c(R, \cdot)$ неотрицательна, $c(R, \xi)=o(\xi)$. Поскольку функция $a(\cdot)$ положительна, то (30) влечет (17) для последовательности $F_{i}=F\left(\cdot ; \lambda_{i}, \tau_{i}\right), \lambda_{i} \rightarrow \infty, \tau_{i} \geqslant 0$. Теперь выполнение условия $\left.\mathbb{B}_{1}\right)$ для данной последовательности $F_{i}$ очевидно.

Для проверки условия $\left.\mathbb{B}_{2}\right)$ положим $E_{0}=C_{0}^{\infty}(\Omega)$. Если $v_{0} \in E_{0}$, то

$$
\mathscr{F}\left(t, v_{0}\right)=v_{0}^{*}+z_{1}(t)
$$

где

$$
\begin{gathered}
v_{0}^{*}=-\operatorname{div}\left(a_{2}\left|\nabla v_{0}\right|^{p-2} \nabla v_{0}\right) \in V^{*} \\
z_{1}(t)=-\operatorname{div}\left(a_{1}(t, \cdot)\left|\nabla v_{0}\right|^{p-2} \nabla v_{0}\right)+b(t, \cdot)\left|v_{0}\right|^{p-2} v_{0} .
\end{gathered}
$$

Множество функций вида $t \mapsto z_{1}(\lambda t+\tau), t \in T, \lambda \geqslant 1, \tau \geqslant 0$, равномерно ограничено в $L^{\infty}\left(T, L^{\infty}(\Omega)\right)$; отсюда следует выполнение условия $\left.\mathbb{B}_{2}\right)$.

Непрерьвность и ограниченность отображения $F_{0}: Y \rightarrow Z$ очевидны. Оценка $(18)\left(\right.$ с $\left.Y_{0}=\Pi\left(T, E_{0}\right)\right)$ также имеет место. Ввиду $\omega$-периодичности функций $a, b$ по $t$ в рассматриваемом случае (18) становится равенством. Таким образом, последовательность $F_{i}=F\left(\cdot ; \lambda_{i}, \tau_{i}\right), \lambda_{i} \rightarrow \infty, \tau_{i} \geqslant 0$, удовлетворяет условию $\left.\mathbb{B}_{3}\right)$.

Проведенные рассуждения означают, что допустимая пара отображений $\mathscr{F}, \mathscr{F} 0$ удовлетворяет условиям $\left.\mathbb{C}_{1}\right)-\mathbb{C}_{3}$ ). Поэтому справедливы теоремы 3,4 . Множество $\mathscr{N}_{0}(h)$ можно рассматривать как совокупность обобшенных решений [1], [9]-[11], [22] начально-краевой задачи

$$
\begin{gathered}
\frac{\partial y}{\partial t}-\operatorname{div}\left(a_{0}|\nabla y|^{p-2} \nabla y\right)+b_{0}|y|^{p-2} y=0 \\
y(\cdot, x)=0, \quad x \in \partial \Omega, \quad y(0, x)=h(x) .
\end{gathered}
$$

Аналогичным образом (с заменой $a_{0}, b_{0}$ на $a(\lambda t+\tau, \cdot), b(\lambda t+\tau, \cdot)$ соответственно) интерпретируется множество $\mathcal{N}(\lambda, \tau ; h)$. Если оператор $\mathscr{F}_{0}$ положительно определен на $V=\stackrel{\circ}{W}_{p}^{1}(\Omega)$, то применима теорема 5, позволяющая находить асимптотику решений класса $\mathcal{N}(\lambda, \tau ; h), \lambda \gg 1, \tau \geqslant 0$, соответствующих задач Коши.

Рассмотренный пример не исчерпывает, разумеется, потенциальных приложений полученных выше результатов. Область их применимости охватывает достаточно широкий класс уравнений (включений) параболического типа. 


\section{Список литературы}

1. Гаевский Г., Грёзер K., Захариас K. Нелинейные операторные уравнения и операторные дифференциальные уравнения. М.: Мир, 1978.

2. Благодатских В.И., Филиппов $A . \Phi$. Дифференциалњные включения и оптимальное управление // Труды МИАН. 1985. Т. 169. С. 194-252.

3. Гельман Б. Д., Обуховский В. В. О новых результатах в теории многозначных отображений. II: Анализ и приложения // Итоги науки и техники. Матем. анализ. Т. 29, 1991. C. $107-159$.

4. Иоффе А.Д., Тихомиров В. М. Теория экстремальных задач. М.: Наука, 1974.

5. Обен Ж. П., Экланд И. Прикладной нелинейный анализ. М.: Мир, 1988.

6. Толстоногов A. A. Дифференциальные включения в банаховом пространстве. Новосибирск: Наука, 1986.

7. Филиппов A.Ф. Диффференциальные уравнения с разрывной правой частью. М.: Наука, 1985.

8. Castaing C., Valadier $V$. Convex analysis and measurable multifunctions. Berlin: Springer-Verlag, 1977. (Lecture Notes in Math. V. 580.).

9. Дубинский Ю. А. Нелинейные эллиптические и параболические уравнения // Итоги науки и техники. Совр. проблемы матем. 1976. Т. 9. С. 5-130.

10. Левитан Б. М., Жииков В.В. Почти-периодические функции и дифференциальные уравнения. М.: Изд-во МГУ, 1978.

11. Лионс Ж.-Л. Некоторые методы решения нелинейных краевых задач. М.: Мир, 1972.

12. Красносельский М.А., Забрейко П. П., Пустыльник Е. И., Соболевский П. Е. Интегральные операторы в пространствах суммируемых функций. М.: Наука, 1966.

13. Климов В.C. К задаче о периодических решениях операторных дифференциальных включений // Изв. АН СССР. Сер. матем. 1989. Т. 53. № 2. С. 309-327.

14. Климов B. С. Эволюционные параболические неравенства с многозначньми операторами // Матем. сб. 1993. Т. 184. №8. С. 37-54.

15. Климов В. С. Об операторе сдвига по траекториям параболических включений // Диффференц. уравнения. 1995. Т. 31. № 10. С. 1716-1721.

16. Климов В. С. Ограниченные решения дифференциальных включений с однородной главной частью // Изв. РАН. Сер. матем. 2000. Т. 64. № 4. С. 109-130.

17. Боголюбов H. Н., Митропольский Ю. А. Асимптотические методы в теории нелинейных колебаний. М.: Наука, 1974.

18. Митропольский Ю. А. Метод усреднения в нелинейной механике. Киев: Наукова думка, 1971.

19. Жиков В.В., Козлов С.М., Олейник О.А. Усреднение дифференциальных операторов. М.: Наука, 1993.

20. Климов B. С. О дифференциальных включениях с однородной главной частью // Дифференц. уравнения. 2002. Т. 38. № 10. С. 1382-1389.

21. Четаев Н. Г. К вопросу об обращении теоремы Лагранжа // Сб. научн. трудов Казанского авиац. инст. 1934. № 2. С. 29-30.

22. Лады женская О. А., Солонников В. А., Уральцева Н. Н. Линейныеи квазилинейные уравнения параболического типа. М.: Наука, 1967.

Ярославский государственный университет

E-mail: igor@alphanet.ru

Поступила в редакцию 26.11 .2002 